\title{
Editorial
}

\section{JNeurosci Manuscripts May Now Include Extended Datasets}

In 2010, JNeurosci made the decision no longer to allow supplemental material because we could not guarantee that the material was sufficiently peer-reviewed. In addition, reviewers sometimes insisted on further analyses and/or experiments that could be added to the supplemental data but did not significantly improve the submitted study. As the years progress, neuroscience papers are becoming more data-rich, and the inability to host material that does not fit in the main body of a manuscript has precluded publication of studies with large datasets in JNeurosci. Providing access to primary data, such as gene ontology lists, protein structures, and chemical compounds, as well as scripts and programs used to analyze data fixed in time to the publication, is essential for many types of neuroscience studies. Hosting these data along with the published version promotes transparency to increase reproducibility of research. To aid this effort, JNeurosci now permits online datasets to be included with submissions in certain circumstances. However, the goal of including Extended Data is not to report supplemental experiments or analyses that the authors are using to support the argument, as those should still be in the main body of the manuscript.

The new policy is outlined below. All Extended Data will be reviewed by the editors to determine whether it is integral to the study and should be included in the final version of the manuscript:

1. If there is a well-established repository for the data, it should be deposited there; JNeurosci will host data without such a repository.

2. Citations may not exist solely within the Extended Data; they must be included in the manuscript. Online datasets are currently not visible to search engines used by citation indices, making inclusion of references in the main manuscript essential to provide proper credit to those cited.
3. Only Extended Data directly related to, or supporting, figures or tables and corresponding figure legends are allowed. Extended Data should be provided in figure or table format and labeled as Figure 1-1, Figure 1-2, Table 1-1, Table 1-2, etc., so they indicate which figure or table they are supporting (i.e. Extended Data table supporting Figure 5 labeled as Figure 5-1). Each should have a legend and be cited in the manuscript text or in the table or figure legend for which they support.

4. For ease of access and review, Extended Data should be submitted in the same format and following the same guidelines as regular figures and tables and uploaded to the system as Multimedia/Extended Data file type (see Figure, Table, Visual Abstract, Multimedia Guidelines).

5. A limitation of $20 \mathrm{MB}$ is recommended. Send an inquiry to jn@sfn.org if larger files are needed.

We are certain that the policy will change as authors provide different types of Extended Data. Our goal is to be flexible, and to address issues as they arise. In particular, the Extended Data policies will have to adapt as new technology develops - both in the types of data and the storage, and presentation capabilities.

Please share this new policy with your colleagues. We are looking forward to hosting increasingly data-rich studies with associated and easily accessed Extended Data. I look forward to getting your feedback at JN_EiC@SfN.org or@MarinaP63 on Twitter.

(D) Marina Picciotto, EiC, JNeurosci

DOI:10.1523/JNEUROSCI.0402-17.2017 